\title{
DERECHO Y POLÍTICAS AMBIENTALES EN LA COMUNIDAD DE MADRID
}

(PRIMER SEMESTRE 2017)

\author{
ANTONIO FORTES MARTÍN \\ Profesor titular de Derecho Administrativo \\ Universidad Carlos III de Madrid
}

Sumario: 1. Subvenciones públicas en las áreas de influencia socioeconómica del Parque Nacional de la Sierra de Guadarrama. 2. Clasificación de la vía pecuaria Cañada Real de Madrid. 3. Ordenación de las actividades piscícolas. 4. Creación del Colegio Profesional de Ambientólogos de la Comunidad de Madrid. 5. Selección de entidad colaboradora para la gestión de ayudas para la adquisición de vehículos eficientes para 2017. 6. Creación del Comisionado del Gobierno de la Comunidad de Madrid para el cambio climático.

\section{SUBVENCIONES PÚBLICAS EN LAS ÁREAS DE INFLUENCIA SOCIOECONÓMICA DEL PARQUE NACIONAL DE LA SIERRA DE GUADARRAMA}

La Orden 2417/2016, de 8 de noviembre, de la Consejería de Medio Ambiente, Administración Local y Ordenación del Territorio, fija las bases reguladoras para la concesión de subvenciones públicas en las áreas de influencia socioeconómica del Parque Nacional Sierra de Guadarrama.

Concretamente, y a partir de lo dispuesto en el artículo 1 de la Orden 2417/2016, el objeto de las subvenciones es la realización de actividades y proyectos en las áreas de influencia socioeconómica del Parque Nacional de la Sierra de Guadarrama de conformidad con lo dispuesto en el Real Decreto 1229/2005, de 13 de octubre, por el que se regulan las subvenciones públicas con cargo a los Presupuestos Generales del Estado en las áreas de influencia socioeconómica de los Parques Naturales. Dichas áreas cabe entenderlas, a partir de lo señalado en el artículo 3.b) de 
la Ley 30/2014, de 3 de diciembre, de Parques Nacionales, como "el territorio constituido por los términos municipales que aportan terreno al mismo, así como, excepcionalmente, siempre que haya causas objetivas que lo definan, por otros directamente relacionados, cuando así se considere en las leyes declarativas, en los que las Administraciones Públicas llevarán a cabo políticas activas para su desarrollo".

Las ayudas se dotan con la finalidad última de promover el desarrollo sostenible de las poblaciones existentes en el área de influencia socioeconómica del Parque Nacional de la Sierra de Guadarrama, en la parte que afecta a la Comunidad de Madrid. En este sentido, entre las actividades subvencionables que se mencionan en el artículo 4 de la Orden $2417 / 2016$ se encuentran todo tipo de iniciativas tendentes a la modernización de las infraestructuras urbanas, periurbanas y rurales destinadas al uso general; las destinadas a la conservación o restauración del patrimonio natural, siempre que presenten un manifiesto valor ecológico; las orientadas a la eliminación de cualquier tipo de impacto sobre los valores naturales o culturales que justificaron la creación del Parque Nacional; las dirigidas a garantizar la compatibilidad de las actividades y usos tradicionales con la finalidad y objetivos del Parque; las destinadas a la conservación o restauración del patrimonio arquitectónico; las que tienen como fin la puesta en marcha de actividades económicas relacionadas con el Parque Nacional de la Sierra de Guadarrama, como las que tienen que ver con la prestación de servicios de atención a visitantes o la comercialización de productos artesanales; las destinadas al mantenimiento o recuperación de la tipología constructiva tradicional de los edificios que constituyen la primera residencia de sus propietarios o tienen un uso directamente relacionado con la actividad productiva asociada al sector primario; las orientadas a la divulgación de los valores e importancia del Parque; las que tienen como misión la formación de la población local en tareas relacionadas con la gestión del Parque Nacional de la Sierra de Guadarrama en cualquiera de sus facetas, con la conservación de los valores naturales y culturales que justificaron su declaración o con el uso sostenible de los recursos naturales renovables; y, por último, las iniciativas 
expresamente previstas en los planes de desarrollo sostenible del Parque Nacional de la Sierra de Guadarrama o en la Agenda 21 de cualquiera de los municipios de la Comunidad de Madrid que conforman sus áreas de influencia socioeconómica.

El resto de la regulación de la Orden 2417/2016 viene referida a aspectos lógicos de las propias bases, sin que sea necesario realizar ningún tipo de consideración más acerca de los potenciales beneficiarios (artículo 3) y sus obligaciones (artículo 5), de las características y cuantía de las ayudas (artículo 6), de la presentación de solicitudes (artículo 8 y 9), del procedimiento de concesión (artículo 10 a 12) o del pago de la subvención (artículo 15), entre otros.

\section{CLASIFICACIÓN DE LA VÍA PECUARIA CAÑADA REAL DE MADRID}

La Orden 2547/2016, de 15 de noviembre, de la Consejería de Medio Ambiente, Administración Local y Ordenación del Territorio, da inicio al procedimiento de clasificación de la vía pecuaria "Cañada Real de Madrid".

El objeto de la Orden es poner fin a una situación que se ha venido prolongando en los últimos años: una vez examinado el Fondo Documental del Área de Vías Pecuarias, de todas las vías pecuarias existentes en Madrid, la Cañada Real es la única que no se encuentra clasificada hasta la fecha mediante la correspondiente Orden Ministerial.

Con la clasificación, en los términos dispuestos por el artículo 7 de la Ley 3/1995, de 23 de marzo, de Vías Pecuarias, y del artículo 13 de la Ley 8/1998, de 15 de junio, de Vías Pecuarias de la Comunidad de Madrid, se determina "la existencia, anchura, trazado, y demás características físicas generales de cada vía pecuaria".

\section{ORDENACIÓN DE LAS ACTIVIDADES PISCÍCOLAS}

Dentro del capítulo relativo a la que hemos dado en llamar ordenación de las actividades piscícolas, pasamos a ocuparnos de la Orden 228/2017, de 13 de febrero, de la Consejería de Medio Ambiente, Administración Local y Ordenación del Territorio, sobre establecimiento de vedas y regulación 
especial de la actividad piscícola en los ríos, arroyos y embalses de la Comunidad de Madrid, para el ejercicio de 2017.

La conservación y regulación de las poblaciones piscícolas que habitan en las masas de agua de la Comunidad de Madrid precisan de su ordenado aprovechamiento de conformidad con lo dispuesto en el artículo 62.1 de la Ley 42/2007, de 13 de diciembre, del Patrimonio Natural y de la Biodiversidad, y del artículo 41.d) de la Ley 1/1983, de 13 de diciembre, de Gobierno y Administración de la Comunidad de Madrid. Y comoquiera que, a partir de las previsiones estatutarias y en virtud del Real Decreto 1703/84, de 1 de agosto, sobre traspaso de funciones y servicios del Estado a la Comunidad de Madrid en materia de conservación de la naturaleza, esta tiene atribuidas las funciones de protección, conservación, fomento y ordenado aprovechamiento de la riqueza piscícola continental y la aplicación de las medidas conducentes a la consecución de estos fines, así como la vigilancia y el control de las aguas continentales, en lo que se refiere a la riqueza piscícola, la Orden objeto ahora de estudio contempla la regulación, para el año 2017, del ejercicio de la pesca deportiva en el ámbito de las aguas de la Comunidad de Madrid.

La disposición no establece una regulación particularmente destacada, por novedosa, de la que se viene aprobando, para cada ejercicio, en años precedentes. Una vez más, el objeto de la Orden es establecer las limitaciones y épocas hábiles para el ejercicio de la pesca en el ámbito de las aguas de la Comunidad de Madrid durante el curso 2017. Más bien, se recrea una ordenación administrativa típica 0 al uso que detalla pormenorizadamente en sus veintiocho artículos: i) los permisos de pesca -en cuanto autorización administrativa que acredita el derecho a ejercitar la pesca en un tramo acotado y en una fecha determinada (y, por tanto, distintos de la licencia de pesca, que el interesado debe tener previamente); a mayor abundamiento, la utilización de estos permisos de pesca determina la clasificación a la que quedan sujetos los pescadores en los términos del artículo 7 de la Orden-; ii) las especies objeto de pesca deportiva en el ámbito territorial de la Comunidad de Madrid (que son las relacionadas en el artículo 9 y anexo I de la Orden) mediante las artes y métodos legales 
(concretados en los artículos 22 y 23 de la Orden, con especificación de los prohibidos en todo el ámbito de la Comunidad de Madrid en el anexo IV de la Orden) en los tramos autorizados a tal fin (donde se diferencian en este punto tramos libres, tramos acotados, tramos de pesca controlada, tramos experimentales de pesca, tramos vedados - art. 13- y, finalmente, un régimen especial de los llamados tramos de captura y suelta -art. 16-); iii) el régimen especial de la pesca en aguas privadas (artículo 17 de la Orden) y el de las zonas piscícolas comprendidas en espacios naturales protegidos y espacios con planes de ordenación de los recursos naturales 0 planes rectores de uso y gestión (artículo 18 de la Orden); iv) las dimensiones mínimas de las especies objeto de pesca, los cupos máximos (ambos extremos también detallados en el citado anexo l y, respectivamente, en los artículos 10 y 11 de la Orden), así como la comercialización y el transporte de las especies objeto de pesca (artículo 26 de la Orden); y, finalmente, y entre otros puntos, v) el régimen de la pesca con fines científicos (artículo 27 de la Orden); y vi) el horario de pesca durante cada jornada, que, de conformidad con el artículo 21, se establece desde una hora antes de la salida del sol hasta una hora después de su puesta. Cabe, no obstante, tener en cuenta que la Consejería de Medio Ambiente, Administración Local y Ordenación del Territorio se encuentra facultada (art. 1.2) para establecer normas de carácter extraordinario cuando sea estrictamente necesario para la conservación de alguna especie de la fauna acuícola continental o bien cuando lo aconsejen los resultados de estudios hidrobiológicos o por cualquier otra causa que por razones de urgencia sea preciso con vistas al establecimiento de dicha normativa extraordinaria.

\section{CREACIÓN DEL COLEGIO PROFESIONAL DE AMBIENTÓLOGOS DE} LA COMUNIDAD DE MADRID

La Ley 3/2017, de 9 de marzo, crea el Colegio Profesional de Ambientólogos de la Comunidad de Madrid. Pese a que no es una ley ambiental stricto sensu, nos permitimos incluirla en la presente crónica por la importancia "ambiental" que reviste la consolidación de una profesión que tiene el medio ambiente como verdadero protagonista de sus actuaciones. 
La propia creación del Colegio Profesional no tiene ninguna particularidad digna de ser destacada. El Colegio Profesional pasa a ser una corporación de derecho público, con personalidad jurídica propia y plena capacidad de obrar, con una estructura interna y un funcionamiento democráticos, y rigiéndose, además, por la Ley 2/1974, de 13 de febrero, sobre Colegios Profesionales, y por la Ley 19/1997, de 11 de julio, de Colegios Profesionales de la Comunidad de Madrid. Ahora bien, interesa tener presente que, conforme a lo dispuesto en el artículo 6 de la Ley 19/1997, de 11 de julio, solo pueden constituirse nuevos colegios profesionales en Madrid respecto de aquellas profesiones cuya aptitud para su ejercicio venga acreditada por la posesión de una titulación oficial y cuando "concurran razones de interés público". Y es justamente la concurrencia de estas razones, en el marco de los valores constitucionales del derecho a un medio ambiente adecuado (artículo $45 \mathrm{CE}$ ) y el derecho a la salud (artículo $43 \mathrm{CE})$, la que mueve a la creación del Colegio Profesional de Ambientólogos, hasta el momento constituidos, en la Comunidad de Madrid, en mera Asociación de Ambientólogos.

En efecto, el propio preámbulo de la Ley 3/2017, de 9 de marzo, llama la atención sobre la "creciente sensibilización y preocupación por el medio ambiente" en los últimos años no solo por parte de los poderes públicos, sino también de la sociedad en su conjunto para poder disponer, en definitiva, de una mayor calidad de vida. La protección ambiental no es solo una cuestión "ciudadana", sino que subyace en ella una dimensión "profesional". De hecho, y tal y como se ha demostrado en las últimas décadas, surge una profesión y unos profesionales que, respondiendo a las demandas sociales y a las exigencias que el ordenamiento jurídico ambiental impone al mundo de la empresa, están llamados, por su formación, a trabajar en busca del continuo equilibrio entre la protección del medio ambiente y el desarrollo económico. Es así como la propia Ley 3/2017, de 9 de marzo, justifica, "desde la perspectiva del interés público, la necesidad de ordenar el ejercicio de esta profesión creando el Colegio Profesional de Ambientólogos de la Comunidad de Madrid, en el que se integren los profesionales que, disponiendo de los conocimientos y la 
titulación necesarios y suficientes, ejerzan esta profesión garantizando el rigor y la calidad de los servicios profesionales que se presten a usuarios y consumidores".

Finalmente, cabe apuntar que el colegio profesional contempla la integración de todos estos profesionales (licenciados o graduados en Ciencias Ambientales), si bien bajo el régimen de adscripción voluntaria. Concretamente, y tal y como prescribe el artículo 4 de la Ley 3/2017, para el ejercicio de la profesión de ambientólogo en Madrid no se requiere la colegiación obligatoria, salvo lo que disponga la legislación básica estatal en la materia. Lejos de constituir un "déficit", la propia Ley aprovecha esta circunstancia para prever un fortalecimiento de la "interlocución con los poderes públicos creando una vía de colaboración con las Administraciones públicas para el óptimo ejercicio de sus funciones que redundará, a su vez, en el fortalecimiento de la protección del medio ambiente en la Comunidad de Madrid".

\section{SELECCIÓN DE ENTIDAD COLABORADORA PARA LA GESTIÓN DE AYUDAS PARA LA ADQUISICIÓN DE VEHÍCULOS EFICIENTES PARA 2017}

La Orden 508/2017, de 15 de marzo, de la Consejería de Medio Ambiente, Administración Local y Ordenación del Territorio, convoca el procedimiento de concurrencia para la selección de entidad colaboradora para la gestión de ayudas para la adquisición de vehículos eficientes para uso de autotaxi para el año 2017. Al mismo tiempo, la Orden 509/2017, de 15 de marzo, de la Consejería de Medio Ambiente, Administración Local y Ordenación del Territorio, convoca, de igual modo, el procedimiento de concurrencia para la selección de la entidad colaboradora para la gestión de las ayudas para la adquisición de vehículos comerciales ligeros eficientes, auxiliares y de servicios para 2017, en los términos dispuestos por los artículos 12 y 16.5 de la Ley 38/2003, de 17 de noviembre, General de Subvenciones.

Ambas órdenes, sobre la base de convocatorias anteriores, asumen el modelo de concesión de la ayuda vía descuento a través de los puntos de venta de los vehículos de que en cada caso se trate, abriendo la 
convocatoria para la selección de la entidad colaboradora encargada de distribuir los fondos a los beneficiarios que cumplan los requisitos establecidos, en el caso de vehículos autotaxi, en la Orden 2157/2013, de 23 de septiembre, de la Consejería de Medio Ambiente y Ordenación del Territorio, por la que se establecen las bases reguladoras para la concesión de las ayudas para la adquisición de vehículos eficientes para uso de autotaxi; y, en el caso de los vehículos comerciales ligeros, en la Orden $3222 / 2014$, de 22 de diciembre, por la que se aprueban las bases reguladoras para la concesión de las ayudas para la adquisición de vehículos comerciales ligeros eficientes, auxiliares y de servicios.

Precisamente, el alcance, tanto de la Orden 2157/2013 como de la Orden $3222 / 2014$, es capital a la hora de continuar apostando por el estímulo para acelerar la renovación de la flota actual de autotaxis y de vehículos comerciales ligeros en la Comunidad de Madrid a través de la incorporación de modelos de alta eficiencia energética y con menor consumo de combustible y menos emisiones de dióxido de nitrógeno y de óxidos de nitrógeno. La apuesta, en este sentido, es por la adquisición de vehículos eléctricos puros, vehículos híbridos, vehículos híbridos enchufables y eléctricos de autonomía extendida, y vehículos de combustión interna altamente eficientes (EURO 6).

En efecto, de resultas de la alta demanda del sector del taxi madrileño y la incidencia que los vehículos comerciales y las flotas de empresas tienen en las emisiones de $\mathrm{NO}_{x}$, sumado al compromiso del Gobierno regional de continuar impulsando la incorporación de vehículos con tecnologías menos contaminantes, la renovación, tanto de la flota actual de autotaxis como de vehículos comerciales ligeros, constituye una de las actuaciones clave para reducir las emisiones a la atmósfera, contemplándose incluso de forma expresa en la Estrategia de Calidad del Aire y Cambio Climático de la Comunidad de Madrid 2013-2020 (Plan Azul +). Máxime cuando las emisiones de $\mathrm{NO}_{x}$ constituyen el principal obstáculo para cumplir los objetivos de calidad del aire establecidos por la UE y, además, el $71 \%$ de las emisiones totales de $\mathrm{NO}_{x}$ en la Comunidad de Madrid provienen del sector del transporte por carretera, lo que incluye los vehículos y las flotas 
de empresas para el desarrollo de la actividad de reparto comercial auxiliar y de prestación de servicios. Por lo que se refiere al sector del taxi, los vehículos para uso de autotaxi representan el 14,8\% de las emisiones contaminantes a la atmósfera pese a suponer solo el $0,5 \%$ del total de vehículos matriculados. Ello se debe a que recorren de media unos 60.000 kilómetros al año, frente a los 15.000 o 20.000 kilómetros al año que realizan de media los vehículos privados. A esto hay que sumar, por otro lado, que esos kilómetros se realizan en su mayoría en un escenario urbano con gran número de arranques y de paradas.

La Orden 508/2017 y la Orden 509/2017 se limitan, así pues, a convocar, en régimen de concurrencia y publicidad, no la concesión de las ayudas mismas, sino el procedimiento de selección de la entidad colaboradora encargada de gestionar las ayudas a la adquisición tanto de vehículos autotaxi como de vehículos comerciales ligeros eficientes. Sujeción a este procedimiento de selección que se justifica por la ausencia de contraprestación económica que conlleva la gestión encomendada. Ello sin perjuicio de la compensación que resulte a favor de la entidad seleccionada (nunca superior al 2,1\% del importe de la convocatoria de ayudas previsto en 2017 para esta línea de ayudas) y con reflejo en el convenio a suscribir por la entidad colaboradora finalmente adjudicataria en los términos dispuestos por el artículo 16 de la Ley 38/2003, de 17 de noviembre, General de Subvenciones.

\section{CREACIÓN DEL COMISIONADO DEL GOBIERNO DE LA COMUNIDAD DE MADRID PARA EL CAMBIO CLIMÁTICO}

El Decreto 36/2017, de 28 de marzo, del Consejo de Gobierno de la Comunidad de Madrid, crea en su artículo 1 el Comisionado del Gobierno de la Comunidad de Madrid para el Cambio Climático como nuevo órgano de la Consejería de Medio Ambiente, Administración Local y Ordenación del Territorio adscrito a la Viceconsejería de Medio Ambiente. El titular del Comisionado pasa a tener la consideración de alto cargo y es nombrado por Decreto del Consejo de Gobierno a propuesta del consejero de Medio Ambiente, Administración Local y Ordenación del Territorio. 
La creación del Comisionado para el Cambio Climático conlleva también, y en lógica consecuencia, una modificación del Decreto 194/2015, de 4 de agosto, por el que se establece la estructura orgánica de la citada Consejería. Concretamente, el artículo 2 del Decreto 36/2017, de 28 de marzo, dispone la creación de un capítulo V en el Decreto 194/2015 para dar cobertura normativa, en el seno de la estructura orgánica de la Consejería de Medio Ambiente, al Comisionado del Gobierno para el Cambio Climático.

La razón que la Comunidad de Madrid arguye para la creación del Comisionado es la de ofrecer un papel más activo en materia de cambio climático, con los consiguientes beneficios ambientales, económicos y sociales. Y, a mayor abundamiento, "centralizar" o canalizar con esta figura las distintas políticas y medidas que se vienen desarrollando en la materia por parte de las diferentes consejerías y centros directivos de la Comunidad de Madrid. De esta forma, y conforme se reconoce en el nuevo artículo 9 del Decreto 194/2015, de 4 de agosto, por el que se establece la estructura orgánica de la Consejería de Medio Ambiente, Administración Local y Ordenación del Territorio, el Comisionado para el Cambio Climático tiene, entre otras, las funciones de coordinación de las diferentes actuaciones de las consejerías de la Comunidad de Madrid en relación con las actuaciones relevantes en la lucha contra los efectos del cambio climático; la asistencia técnica y el asesoramiento a los diferentes centros directivos de la Administración autonómica; la coordinación de los foros de participación y coordinación que se constituyan en el seno de la Administración autonómica; el impulso y participación en la revisión de la Estrategia de Calidad del Aire y Cambio Climático de la Comunidad de Madrid; y, finalmente, la representación de la Comunidad de Madrid en materia de cambio climático en sus relaciones con otras administraciones públicas, instituciones públicas y privadas, y ONG. 